\title{
Psychiatrie im dritten Jahrzehnt des 21. Jahrhunderts
}

\author{
Korrespondenzadresse \\ Prof. Dr. Dr. Manfred Spitzer \\ Universität Ulm \\ Abteilung für Psychiatrie \\ Leimgrubenweg 12-14 \\ 87054 Ulm, Deutschland
}

\author{
Bibliografie \\ DOI https://doi.org/10.1055/a-1246-0345 \\ Nervenheilkunde 2021; 40: 6-12 \\ (C) 2021. Thieme. All rights reserved. \\ Georg Thieme Verlag KG, Rüdigerstraße 14, \\ 70469 Stuttgart, Germany \\ ISSN 0722-1541
}

Wenn dieses Editorial erscheint, befinden wir uns im dritten Jahrzehnt des 21. Jahrhunderts. Was bedeutet das für unser Fach? Welche Entwicklungen zeichnen sich ab? Leider habe ich keine Glaskugel zur Hand, und selbst wenn ich eine hätte, so verfüge ich dennoch über keinerlei hellseherischen Fähigkeiten. Aber es gibt viele neue Erkenntnisse, Entwicklungen und gewisse Trends schon jetzt, die man auf die Psychiatrie extrapolieren ${ }^{1}$ kann: Molekularbiologie, Gentechnik, Epigenetik, Mikrobiom, Data-Science, der Wandel von Demografie und Klima, die Verstädterung oder die ubiquitäre Verbreitung von Internet und digitalen Endgeräten. All das wird nicht nur ganz sicher die Oberflächengestalt psychopathologischer Erscheinungen verändern (in dem Sinne, wie der hypochondrische Wahn Depressiver früher die Lues, dann TBC, dann HIV und demnächst wahrscheinlich Corona zum Inhalt hat oder im Sinne von Phantomvibration, Fomo und Cyberchondrie), sondern mit hoher Wahrscheinlichkeit tiefer gehen: So wie der Abstammungswahn mit dem Adel (und etwa 100-jähriger Verspätung) verschwand, könnten bestimmte Formen der Psychopathologie ab- oder zunehmen: Dass der demografische Wandel mehr demenzielle Syndrome mit sich bringen wird, ist trivial; dass der Klimawandel mehr Ängste und Gefühle der Hilflosigkeit (und damit auch mehr Depression) zur Folge haben wird, ist schon nicht mehr ganz so trivial. Und dass manche klinische Einschätzung irgendwann auch in der Psychiatrie durch Data-Science ersetzt wird, oder Psychotherapie durch ein Klistier, ist zumindest nicht mehr prinzipiell undenkbar. Betrachten wir ein paar Beispiele - in leider noch ungeordneter Reihenfolge im Hinblick auf Inhalt und Wahrscheinlichkeit des Eintretens. ${ }^{2}$

\section{Gene-Editing: CRISPR/Cas bei Alzheimer?}

Die Alzheimer-Krankheit macht etwa $70 \%$ der Demenzen aus und gehört mit knapp 50 Millionen Fällen weltweit zu den häufigen Todesursachen. Bereits in 10 Jahren werden es über 70 Millionen Fälle weltweit sein. Bei ihr kommt es u. a. zu einem „falschen Zerschneiden“ eines in der Zellmembran lokalisierten Eiweißkörpers, dem

1 Gemeint ist hier am ehesten die juristische Extrapolation, die den Schluss von „A impliziert B“ und „B impliziert C“ auf „A impliziert C“ meint.

2 Die Zeit der Abfassung dieses Textes - zwischen drittem und vierten Advent, mit immer mehr Corona-Infizierten und -Toten und mit Lockdown - erscheint geradezu prädestiniert für solcherart Brainstorming.
Amyloid-Precursor-Protein (APP). Hierbei entsteht Amyloid, dessen Ablagerung im Gehirn für die Krankheit mitverantwortlich ist. In einer isländischen Studie an 1700 Patienten wurde bereits im Jahr 2012 eine Mutation im APP-Gen entdeckt, die mit der Abwesenheit von Alzheimer-Demenz assoziiert war [15]. Diese Mutation betrifft ein einziges Nukleotid (Punktmutation), das vor kurzem erstmals mittels der Genschere CRISPR/Cas in Zellkulturen eingesetzt wurde, woraufhin sich die Ablagerungen verminderten [19]. Die ganze Sache klingt sehr gut, ist jedoch aus ganz prinzipiellen Gründen nicht unproblematisch: Zwar nimmt man an, dass die bei den Isländern gefundene Mutation sonst keine negativen Auswirkungen hat. Das kann sein. Nicht selten haben aber Gene (um genau zu sein: Allele), die für irgendetwas gut sind, irgendwo anders negative Auswirkungen, die man (zunächst) meist nicht kennt. 3 bekannte Beispiele: Gene für blaue Augen und helle Haut vermindern in höheren Breitengraden Vitamin-D-Mangel im Winter, führen jedoch in Gegenden mit mehr Sonneneinstrahlung (also näher am Äquator) zu mehr Hautkrebs. Gene für besonders gutes Denkvermögen in jungen Jahren erhöhen die Wahrscheinlichkeit der Entwicklung einer Alzheimer-Demenz im Alter. Gene für ein langes Leben erhöhen die Wahrscheinlichkeit, an Krebs zu erkranken, denn Krebszellen vermehren sich, leben ewig und führen daher zum Tod.

\section{Darm-Gehirn-Achse: Bakterien gegen Angst, Autismus und Depression?}

Wohl kaum ein Thema hat die Medizin im vergangenen Jahrzehnt so stark und so unerwartet durcheinandergewirbelt wie das Mikrobiom, also die auf und in unserem Körper vorhandenen Bakterien. Dass Kot zu etwa $70 \%$ aus Bakterien besteht, wusste man schon lange, dass diese Bakterien jedoch in ihrer Summe mehr als das 100-Fache der Gene des menschlichen Körpers ausmachen und damit Stoffwechselleistungen vollbringen können, die wir nicht können, war neu. So können wir keine Fasern verdauen, unser Mikrobiom aber schon. Und es macht daraus nicht nur etwa ein Zehntel der Kalorien, die wir täglich zu uns nehmen, sondern auch Stoffe, die wir nicht herstellen können: Manche Vitamine und beispielsweise kurzkettige Fettsäuren, die für die Gehirnentwicklung gebraucht werden.

In gleich 3 unterschiedlichen Mausmodellen des Autismus konnte gezeigt werden, dass die Übertragung von gesunden Darmbak- 
terien (Lactobacillus reuteri) in den Darm der Modell-Tiere die Entstehung dieser Entwicklungsstörung verhindert [23]. Bemerkenswert an den Experimenten war die Tatsache, dass der Autismus durch die Bakterien sowohl bei gleichzeitiger Durchtrennung des Nervus vagus als auch bei Blockade zerebraler Oxytocin-Rezeptoren nicht zu verhindern war. Auch die Blockade dopaminerger Verbindungen der VTA hatte diese Auswirkung. Der therapeutische Effekt der Darmbakterien ist also über den Vagusnerv sowie über die zentralnervösen Neurotransmitter Oxytocin und Dopamin vermittelt ( $\triangleright$ Abb. 1). An diesem Beispiel zeigt sich deutlich die Komplexität der Auswirkungen der „richtigen“ Darmbakterien auf die Gehirnentwicklung.

- Für den Effekt muss es ein „Entwicklungsfenster“ (auch „kritische Phase“ oder „sensible Periode“ genannt) geben.

- Er setzt die Intaktheit des Parasympathikus und gleich zweier für Lernen und Sozialverhalten als wesentlich erachteter Neuromodulatoren voraus.

- Der Effekt erwies sich als unabhängig von der Ursache des Autismus.

Das Beispiel zeigt, wie unerwartet die Ergebnisse gut gemachter wissenschaftlicher Studien sein können. Es zeigt aber auch, dass hier lediglich ein Anfang gemacht wurde und noch sehr großer Forschungsbedarf besteht, bis diese Erkenntnisse klinische Relevanz bekommen könnten. Auch Angststörungen und Depression wurden mit Veränderungen des Mikrobioms im Darm des Menschen in Verbindung gebracht $[22,37]$. Die Neuromodulatoren Serotonin, Dopamin, Noradrenalin, Melatonin sowie die Neurotransmitter Acetylcholin, Glutamat und GABA - das muss jeden Nervenarzt aufhorchen lassen - werden nebst den genannten kurzkettigen Fettsäuren und Vitaminen vom Mikrobiom in unserem Darm für uns synthetisiert ( $\triangleright$ Abb. 2).

In Mausmodellen führt die Einnahme von Probiotika (also Stoffen, welche die Darmbakterien beeinflussen) durch Muttertiere während der Trächtigkeit und Laktation zu messbaren Veränderungen bei der Expression bestimmter Neurotransmitter sowie angstspezifischer Verhaltensweisen bei den Nachkommen [12]. Diese Befunde zeigen, dass das die mütterliche Diät epigenetische Effekte auf das Gehirn der Nachkommen haben kann, die über das Mikrobiom vermittelt sind. Die klinischen Konsequenzen dieser Befunde sind Gegenstand intensiver Forschungsbemühungen und werden uns damit in den nächsten Jahren mit hoher Wahrscheinlichkeit in der Klinik beschäftigen. Da Bakterien sich in einer Matrix aus Zellulose verkapseln lassen, ist deren Einsatz neben dem von Probiotika auch in der Psychiatrie durchaus denkbar. Es muss also keineswegs um „fecal transplants“ gehen, wie man das im Englischen nennt, also Klistiere mit Bakterien aus den Därmen Gesunder.

\section{Big Data, Mustererkennung und klinische Entscheidungen}

Die Analyse großer Datensätze mittels lernender Computer, die ähnlich funktionieren wie lernende Gehirne, erobert seit ein paar Jahren die unterschiedlichsten Bereiche unserer Welt - autonom fahrende Autos, automatisches Übersetzen und Erkennen von Gesichtern, Wettervorhersage, Auffinden neuer chemischer Reakti-

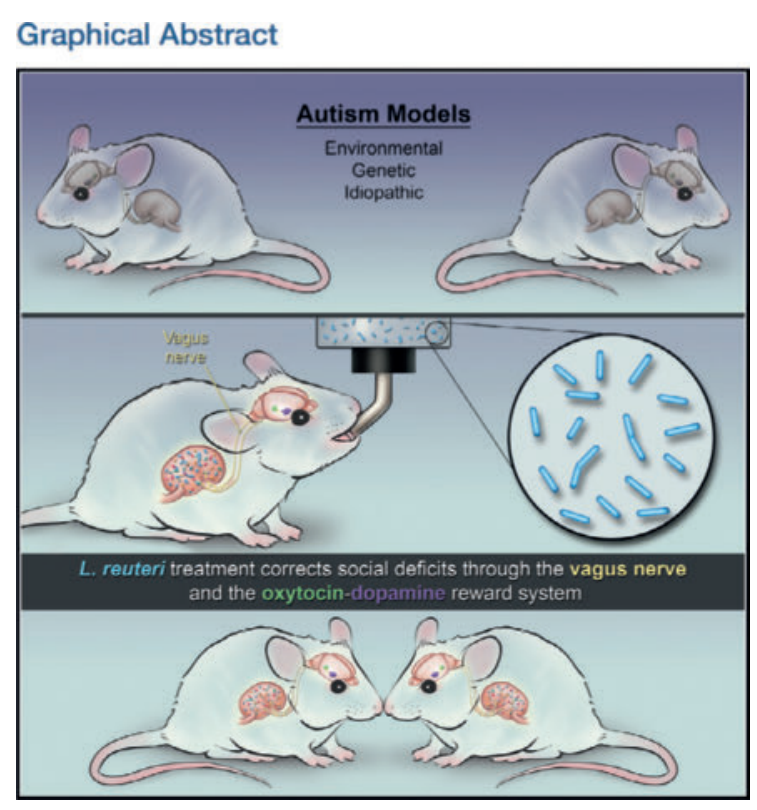

- Abb. 1 Diese besonders nette grafische Zusammenfassung der Studie aus Neuron sei dem Leser nicht vorenthalten (nach Daten aus [23]).

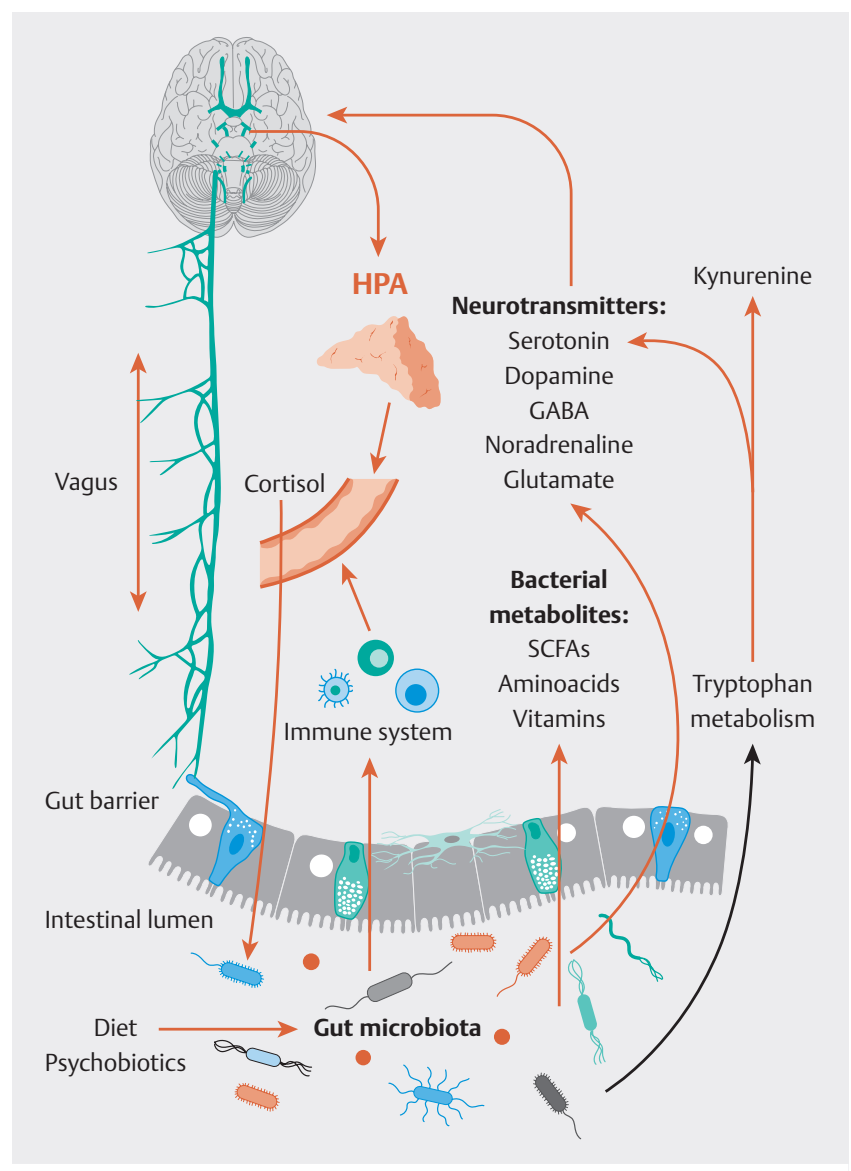

Abb. 2 Zusammenhang auf der molekularen bzw. Neurotransmitterebene zwischen Darmbakterien und Depression (nach Daten aus [4]). 


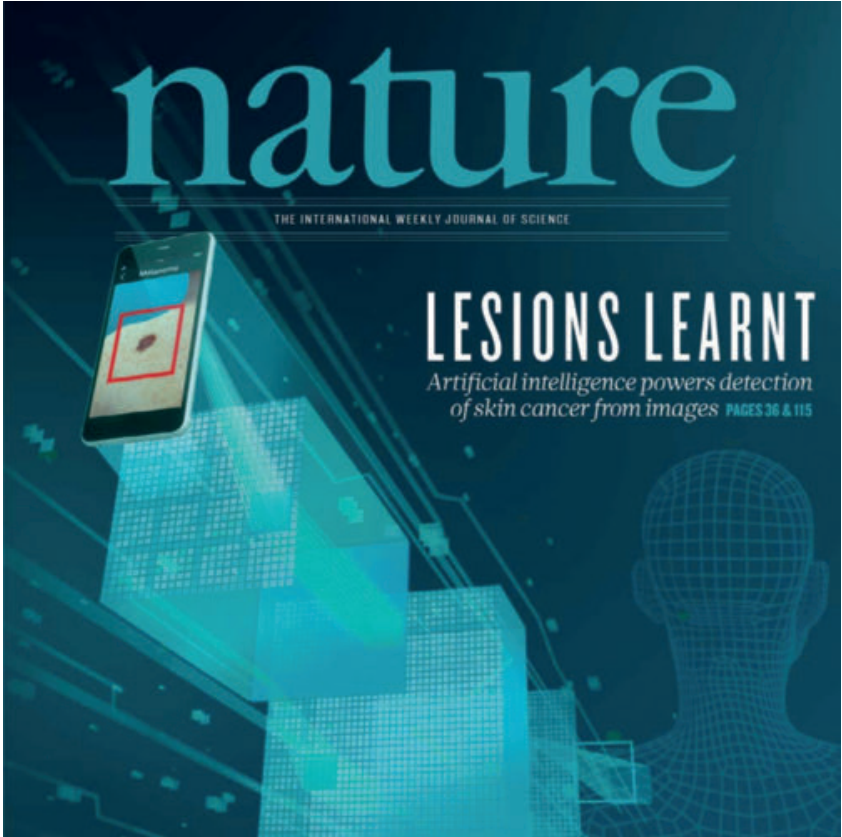

- Abb. 3 Cover des Nature-Hefts aus dem Jahr 2017, auf dem der Durchbruch der Nutzung von Artificial Intelligence (AI) bei der medizinischen Diagnose von Hautkrebs sehr schön in grafische Symbolik umgesetzt wurde. Auch der Titel „Lesions Learnt“ ist kein Druckfehler, sondern stellt ein schönes Wortspiel dar, werden doch normalerweise „lessons“ gelernt. In diesem Fall hat das neuronale Netzwerk jedoch bei Hautläsionen („lesions“) gelernt, die gutartigen von den bösartigen zu unterscheiden.

onswege $^{3}$ - und auch die Medizin in vielfacher Hinsicht. Der wesentliche Fortschritt bestand dabei in immer stärkerer Rechenleistung von Computern und immer besseren Algorithmen zum Lernen (Deep Learning). Mein Smartphone kann, verbunden mit dem Internet, Hautkrebs so gut diagnostizieren wie ein Dermatologe [7] ( Abb. 3). Computer sind bei der Beurteilung beispielsweise von Lungen-CTs zum Screening für Lungenkarzinomen etwa so gut wie Radiologen [3] und werden in naher Zukunft Radiologen auch bei der Beurteilung von Röntgenbildern ablösen (ein Computer und ein Radiologe zusammen sind ebenso gut oder gar besser als 2 Radiologen ${ }^{4}, 5$ ). Mittels künstlicher Intelligenz (Deep Learning in neuronalen Netzwerken) finden Maschinen mittlerweile sogar neue Erkenntnisse, auf die Radiologen nie gekommen wären (

3 "Als will not replace chemists, [...] but the chemists that use Als will replace the chemists that don't“ zitiert Joshua Howgego [14] den Chemiker Derek Lowe einer pharmazeutischen Firma in Massachusetts (in deutscher Übersetzung: „Künstliche Intelligenzen werden Chemiker nicht ersetzen, aber Chemiker, die künstliche Intelligenz verwenden, werden Chemiker ersetzen, die sie nicht verwenden“).

4 "Al won't replace radiologists, but radiologists who use Al will replace radiologists who don't," zitiert Reardon [24] den Radiologen Curtis Langlotz von der Stanford University (in deutscher Übersetzung: „Die künstliche Intelligenz wird Radiologen nicht ersetzen, aber Radiologen, die künstliche Intelligenz verwenden, werden Radiologen ersetzen, die sie nicht verwenden“).

5 Man beachte die Ähnlichkeit der beiden Zitate in den Anmerkungen 3 und 4. Frei nach dem Motto „einmal ist keinmal, zweimal ist immer“ kann man vermuten, dass das Zitat sinngemäß nicht nur in der Chemie und der Radiologie gilt.
Was man mit der Analyse von Daten aus der realen Welt durch lernende neuronale Netzwerke in der Medizin bei der klinischen Entscheidungsfindung anstellen kann, zeigte schon vor mehr als 2 Jahrzehnten der Arzt einer Unfallklinik im amerikanischen Bundesstaat North Carolina [25, 30]. Er trainierte ein neuronales Netzwerk mit Daten zu Alter, Geschlecht, Diagnose, klinischem Bild, Allgemeinzustand, Laborwerten und Outcome (überlebt oder gestorben) von Patienten auf Intensivstationen, sodass es lernte, aus diesen Datenmustern die Prognose der Patienten vorherzusagen. Dies ist wichtig, wenn es beispielsweise um die Triage geht, $d$. h. um die Optimierung des Outcomes bei der Allokation knapper Ressourcen: Wenn nach einer Massenkarambolage 7 Schwerverletzte zugleich eintreffen, aber nur 4 Intensivbetten zur Verfügung stehen, sollte die Entscheidung, wer behandelt wird und wer nicht, nach rationalen Gesichtspunkten getroffen werden. ${ }^{6}$ Gelöst wird es in aller Welt nach den gleichen Prinzipien. Wenn an der Lösung künstliche Intelligenz beteiligt ist (kein Mensch kann so viele Daten wie neuronale Netzwerke verarbeiten und dies ohne affektiv getönte und wertende (Vor-)Urteile), sagen die Amerikaner „der Computer entscheidet“, wohingegen man in Europa eher sagt, „dass wir entscheiden, uns aber vom Computer unterstützen lassen“.

Erst kürzlich wurde eine auf maschinellem Lernen basierte Lösung des bekannten Problems publiziert, das bei der Gabe von Antibiotika bei einem unkomplizierten Harnwegsinfekt dadurch entsteht. Man will einerseits wirksam therapieren, andererseits jedoch keineswegs immer gleich das neueste und wirksamste Antibiotikum geben, um die Entwicklung von Resistenzen gegen diese neu entwickelten Substanzen nicht zu fördern [17]. Welches Medikament sollte man also vor dem Hintergrund dieses Zielkonflikts zwischen jetzigen und künftigen Patientinnen im Einzelfall geben? Dieses Problem ist keineswegs akademisch, sondern im klinischen Alltag sehr häufig, erleidet doch nahezu jede Frau mindestens einmal im Leben einen Harnwegsinfekt, welcher mit in den USA 4,7 Millionen Verschreibungen bei 13 Millionen ambulanten Vorfällen die dritthäufigste Indikation für die Gabe von Antibiotika überhaupt darstellt. Um das Problem zu lösen, entwickelte ein Team aus Ärzten und Wissenschaftlern aus Boston (Harvard University und Medical School, MIT) und Pittsburgh (Machine Learning Department der Carnegie Mellon University) eine Anwendung, um auf der Grundlage von maschinellem Lernen aus Daten der elektronischen Gesundheitsakte (EHR), sowohl Wirksamkeit als auch Wahrscheinlichkeit einer Antibiotikaresistenz gegen Erstlinien- (Nitrofurantoin und Trimethoprim-Sulfamethoxazol) und Zweitlinien-Therapien (Ciprofloxazin und Levofloxazin) vorherzusagen. Der daraus entwickelte Entscheidungsalgorithmus setzt diese Wahrscheinlichkeiten in Empfehlungen um, sodass das Antibiotikum mit dem kleinstmöglichen Spektrum ausgewählt wurde, mit dem sich noch eine klinische Heilung erreichen lässt. In den Jahren 2014-2016 wurde dann bei einer Testkohorte von 3629 Patienten eine Verminderung der Verwendung von Zweitlinien-Therapien um $67 \%$ gegenüber der Entscheidung von Klinikern gefunden. Zugleich wurden Behand-

$6 \quad$ Wegen der knapp werdenden Betten bzw. des knapp werdenden Personals auf Intensivstationen unter der laufenden Corona-Pandemie hat dieses Problem in den vergangenen Wochen und Monaten wieder viel Aufmerksamkeit erlangt. Es ist so alt wie die Militärmedizin, denn in Kriegen stellt es sich häufig. 

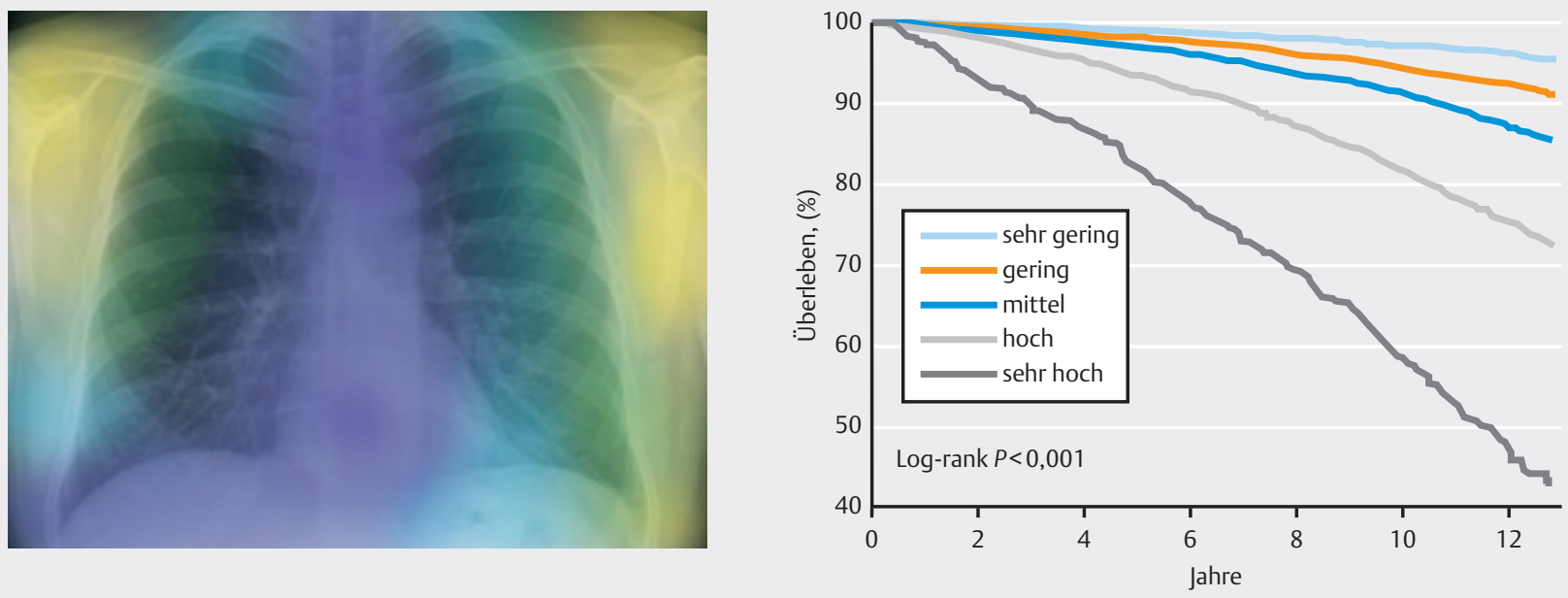

- Abb.4 Die Stellung der Schulterblätter im Röntgenthorax verrät etwas über die Mortalität (nach Daten aus [21]). Zu diesem Ergebnis kommt eine Studie an 52320 Röntgenthorax-Aufnahmen, die im Rahmen eines Screening-Programms zur Vorsorge im Hinblick auf Karzinome von Prostata, Lunge, Kolon und Rektum sowie der Ovarien vom 8. November 1993 bis zum 2. Juli 2001 an 10 Zentren in den USA durchgeführt wurden. Ein neuronales Netzwerk wurde dann mit nichts weiter trainiert als den Röntgenbildern und den Sterbedaten der Teilnehmer in den darauffolgenden 12 Jahren. Die Abbildung links zeigt anhand einer Beispiel-Röntgenaufnahme farblich kodiert (von Violett: „wenig“ bis Gelb: „viel“) die Lokalisation der Varianzaufklärung, d. h. zeigt an, dass das Netzwerk die Mortalität vor allem an den Schulterblättern abgelesen hat. Teilt man die Gesamtgruppe nach der vorhergesagten Mortalität in fünf Gruppen (Quintile) ein, so zeigen die Kaplan-Meier-Überlebenskurven (rechts) deutliche Unterschiede in der 12-Jahres-Mortalität zwischen den Gruppen an. Zur Validierung wurde das Netzwerk danach zur Bestimmung der 6-Jahres-Mortalität anhand weiterer 5493 Röntgenthoraxbilder aus einer anderen Studie (vom August 2002 bis zum April 2004) verwendet und sagte diese gut voraus. Die Voraussage funktionierte unabhängig von der Diagnose im Einzelfall. Warum das so ist, weiß bislang niemand. (Und dies ist bei lernenden neuronalen Netzwerken, die nach dem Lernen etwas können, grundsätzlich so: Wie sie das machen, ist unserer Erkenntnis zunächst verschlossen. Man kann auf verschiedene Weise versuchen, es herauszubekommen, aber die Erfolgsaussichten sind ungewiss.)

lungen mit Antibiotika, gegenüber denen sich die Erreger als resistent erwiesen, um $18 \%$ durch den Algorithmus (gegenüber Klinikern) gesenkt. In $92 \%$ (1066 von 1157) der Fälle, in denen Kliniker eine Zweitlinien-Therapie empfohlen hatten, der Algorithmus jedoch ein Erstlinien-Medikament vorschlug, erwiesen sich die Erreger als empfindlich auf das Erstlinien-Medikament. Wenn Kliniker ein ungeeignetes Medikament der ersten Wahl auswählten, wählte der Algorithmus in 47 \% (183 von 392) der Fälle ein geeignetes Medikament der ersten Wahl. Die Autoren folgern, dass ihr „maschinell lernender Entscheidungsalgorithmus Antibiotikaempfehlungen für ein häufiges infektiöses Syndrom bietet, indem er die Reduktion des Einsatzes von Breitbandantibiotika bei gleichzeitiger Aufrechterhaltung optimaler Wirksamkeit gewährleistet" [17]. Sie sind damit nicht am Ende, sondern erst am Anfang ihrer Arbeit, denn erst weitere solche Untersuchungen mittels neuronaler Netzwerke, die aus klinischen Daten Muster extrahieren und damit Handlungsanweisungen optimieren, wie es ein einzelner Kliniker schlichtweg nicht kann7, können die Verallgemeinerbarkeit ihrer Ergebnisse auf andere Diagnosen und Patientengruppen klären.

Was hat das Ganze mit Psychiatrie zu tun? - Sehr viel, wie ein Blick in die Psychiatriegeschichte zeigt. Diese ist voll von Beispielen der Erkennung von Mustern, auch wenn die beteiligten Psychi-

$7 \quad$ Klinische Erfahrung ist in dieser Hinsicht nichts anderes als die Spieleerfahrung eines guten Poker-, Schach- oder Go-Spielers. Bekanntermaßen wurde für diese Spiele vor Jahren schon gezeigt, dass Maschinen sie besser beherrschen als Menschen und daher gewinnen. Sie können einfach schneller und viel mehr lernen [31]. ater manchmal Muster zu erkennen glaubten, die sich als ephemer herausstellten. So beispielsweise in der zu Beginn des 19. Jahrhunderts von dem Arzt und Anatomen Franz Joseph Gall (1758-1828) begründeten Phrenologie, in der man aus bestimmten Mustern der Form des Schädels auf Charaktereigenschaften schließen zu können glaubte $(\triangleright \mathbf{A b b} \mathbf{~} \mathbf{5})^{8}$, oder darauf aufbauend einige Jahrzehnte später in den Lehren von Cesare Lombroso (1872) zu Gesichtszügen von „Degenerierten“ und Verbrechern. ${ }^{9}$ Auch Sir Francis Galton (1878, 1879), ein Neffe von Charles Darwin, versuchte sich in der Erkennung des „typischen“Verbrechergesichts durch fotographische Mittelwertbildung. Bleibenden Erfolg hatten dagegen die Psychiater Emil Kraepelin (1890), der psychiatrische Erkrankungen nach ihren Verlaufsmustern einteilte, Eugen Bleuler (1911), der Muster

8 Diese topologisch ausgerichtete Lehre versuchte, geistige Eigenschaften und Zustände zu bestimmten, klar abgegrenzten Hirnarealen zuzuordnen. Dabei wurde ein Zusammenhang zwischen Schädel- und Gehirnform einerseits und Charakter und Geistesgaben andererseits unterstellt. Die Bezeichnung dieses biologischen Ansatzes in der Charakterlehre als Phrenologie erfolgte ab 1815 auf Vorschlag des englischen Naturforschers Thomas Forster. In der ersten Hälfte des vorletzten Jahrhunderts war diese Theorie in Europa und im angloamerikanischen Raum weit verbreitet und sehr bekannt. Im Jahr 1820 wurde in Schottland die „Edinburgher Phrenologische Gesellschaft“ gegründet, die ab 1823 eine eigene Zeitschrift veröffentlichte. Zu den Vertretern der Theorie in den USA zählten Orson Squire Fowler und Lorenzo Niles Fowler, der später nach England ging und dort 1887 die „Britische Phrenologische Gesellschaft“ gründete, die bis 1967 fortbestand.

9 Zur psychiatriegeschichtlichen Bedeutung der Degenerationslehre von Lombroso $[13,30]$. 


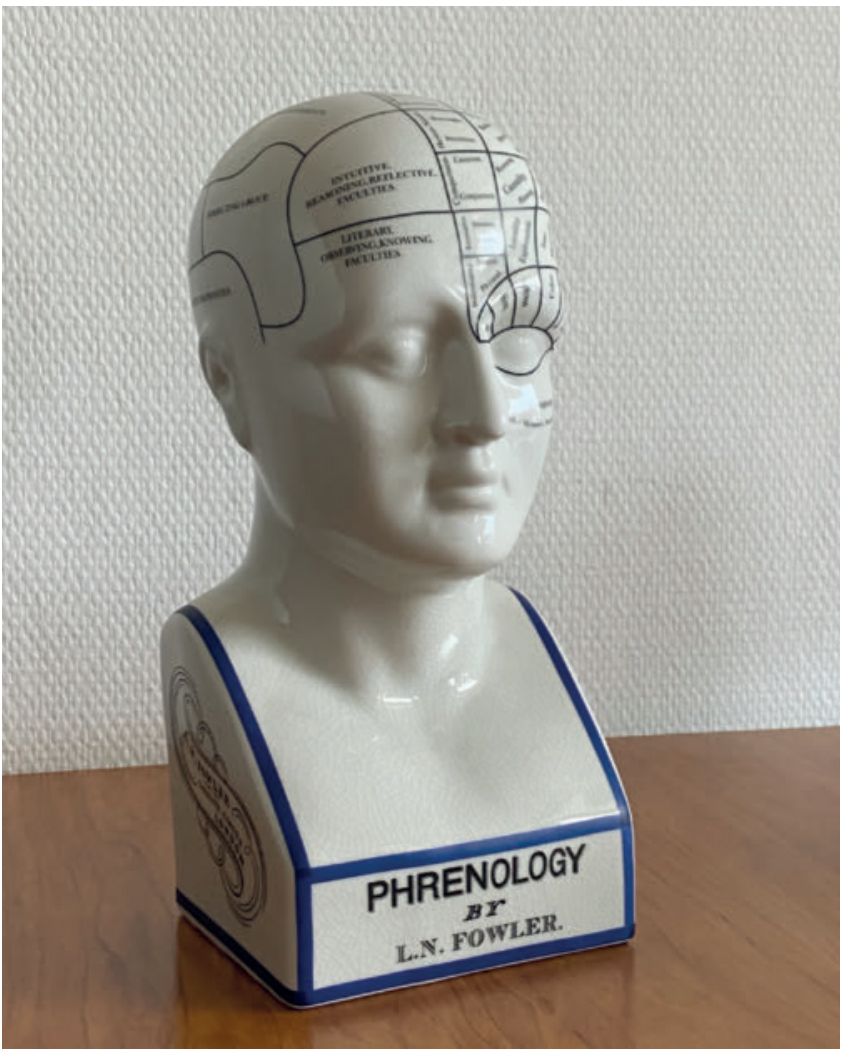

- Abb.5 Die vielleicht bekannteste Büste zur Phrenologie stammt von Lorenzo Niles Fowler (Foto: @Autor).

von Abweichungen normaler geistiger Prozesse (Denken, Affekt, Wollen und Sozialverhalten) in seinen Grundsymptomen der Schizophrenie beschrieb, sowie Kurt Schneider (1946/1980), der ganz bestimmte klinische Muster (das Hören von kommentierenden oder dialogisierenden Stimmen, Gemachtes, „2-gliedrige“ Wahnwahrnehmungen) als Erstrangsymptome der Schizophrenie beschrieb. Einflussreich, aber letztlich ohne langfristige klinische Bestätigung blieben auch die Versuche des tübinger Psychiaters Ernst Kretschmer (1927), aus 3 idealtypischen Mustern des Körperbaus auf bestimmte damit korrelierende Charaktereigenschaften zu schließen.

Die genannten Psychiater würden heute angesichts der mittlerweile sehr erfolgreichen Versuche, durch maschinelles Lernen Millionen von Mustern jeglicher Art (Gesichter, zeitliche Verläufe, semantische Beziehungen, Straßenverkehrsschilder und -teilnehmer, chemische Substanzen, Krankenakten, Röntgenbilder, Eiweißkörper, Gerüche etc.) zu erkennen und zu klassifizieren, wahrscheinlich sofort die „Europäische Gesellschaft für Computational Psychiatry“ gründen! Ähnlich wie man darüber nachdenken kann, wie oder was Mozart oder Beethoven heute komponieren würden, kann man fragen, ob Kraepelin heute mit Hilfe geeigneter Software und Smartphone-Daten zum Verlauf von Aktivität, Denken, Affekt, Suizidalität, Vorlieben, Kommunikation mit anderen und zur Persönlichkeit Datenbanken erstellen würde, um diese dann mittels Deep Learning klassifikatorisch zu analysieren. Außenkriterien wie erreichter Bildungsgrad, Einkünfte, Ausgaben, Beziehungsstatus und Güte des sozialen Netzwerks könnte er sich von den Rechenzentren von Facebook, Amazon, Apple und Google besorgen, wo man sie sowieso längst analysiert hat [32].

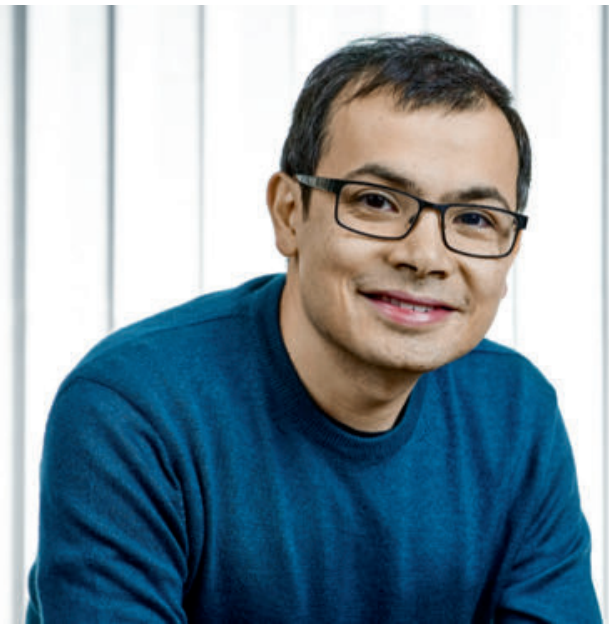

- Abb. 6 Demis Hassabis, der Gründer und noch immer die treibende Kraft der Firma DeepMind. Am 14.11.2017 hielt er auf der Jahrestagung der Society of Neuroscience - wie jedes Jahr trafen sich etwa 30000 Gehirnforscher zum Auszutausch - eine Keynote Rede. Vor Tausenden Neurowissenschaftlern sagte er lässig: „Erst lösen wir das Problem, was Intelligenz ist, und wenn wir das gelöst haben, dann lösen wir alle anderen Probleme." Hätte er zu diesem Zeitpunkt nicht schon bahnbrechende Erfolge verbuchen können, hätten die Kollegen ihn wahrscheinlich nicht wirklich ernst genommen. So aber war es sehr still im Saal als er der versammelten Mannschaft der Neurowissenschaftler dieser Welt klarmachte, dass Artificial Intelligence (Al) im Grunde nichts weiter ist als angewandte Gehirnforschung, wie er dies im Fachblatt Neuron publiziert hat. Quelle: Foto: @ DeepMind

\section{Big Data und neue Medikamente}

Die Firma DeepMind wurde im Jahr 2010 durch den Neurowissen-

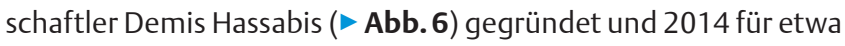
400 Millionen Englische Pfund an Google (heute Alphabet) verkauft. Sie hat mittlerweile mehr als 1000 Mitarbeiter (Stand: 20.11.2020), was nicht weiter verwundert, ist doch ihr erklärtes Ziel, zunächst Intelligenz zu verstehen, um dann alle anderen Probleme dieser Welt zu lösen. Ihr erster großer Erfolg bestand darin, dass das von DeepMind entwickelte selbstständig anhand von Millionen publizierter Go-Partien lernende neuronale Netzwerk AlphaGo im Jahr 2016 gegen den besten Go-Spieler der Welt mehrfach gewonnen hatte [28]. Ein Jahr später war ein Nachfolgemodell - AlphaGo Zero - entwickelt worden, das von Anfang an nur gegen sich selbst spielte (also nicht von Menschen lernte) und nach 40 Tagen Training 100 von 100 Partien gegen AlphaGo gewann [29]. Die Methodik des Deep Learning taugt jedoch zu weit mehr als nur zum Spielen, kann man mit ihr doch beispielsweise auch neue Medikamente entdecken. Der Hintergrund hierzu lässt sich wie folgt in aller gebotenen Kürze darstellen.

Der Chemiker Christian B. Anfinsen ( $\bullet$ Abb. 8 ) hatte bereits im Jahr 1972 in seiner Rede anlässlich des Empfangs des Nobelpreises für Chemie gefordert, dass man - zumindest theoretisch und im Prinzip - die 3-dimensionale Struktur eines Proteins aus der eindimensionalen Abfolge von dessen Aminosäuren vorsagen können müsste. Das klingt logisch, wurde jedoch damals zugleich als unmöglich erachtet. Denn bereits im Jahr 1969 hatte der US-amerikanische Molekularbiologe Cyrus Levinthal darauf hingewiesen, 
dass die Zahl der Möglichkeiten der Faltung eines relativ kleinen Eiweißkörpers (aus 150 Aminosäuren) $2^{150}$ beträgt (entspricht etwa $1,4 \times 10^{45}$ ), wenn man hierzu noch vereinfachend annimmt, dass jede Aminosäure nur 2 Zustände einnehmen kann. Bei größeren Proteinen kann die Zahl $10^{300}$ übersteigen, also eine Zahl, die in der Natur nicht vorkommt, wird doch die Zahl der Atome im gesamten Universum auf etwa $10^{80}$ geschätzt (man spricht daher auch vom Levinthal-Paradoxon ${ }^{10}$ ).

Mit Hilfe einer Proteindatenbank lernte AlphaFold anhand von Millionen von Beispielen die Form eines Proteins aus der Reihenfolge seiner Aminosäuren zu etwa 60 \% vorherzusagen, wie im Januar 2020 im Fachblatt Nature publiziert wurde [27]. Die Genauigkeit betrug im Mittel 6,6 Ångström, was noch nicht ausreicht, um die katalytischen Effekte von Proteinen anhand der räumlichen Passungen zwischen Proteinen und Stoffen, die deren Wirkungen beeinflussen (d. h. die meisten Medikamente!) vorherzusagen. Hierfür wird eine Auflösung von 2-3 Ångström benötigt [1]. Es ist daher von großer Bedeutung, dass die Anfang Dezember (vorab) publizierte neueste Version des die Proteinfaltung vorhersagenden neuronalen Netzwerks - genannt AlphaFold2 - Ergebnisse mit einer Auflösung von 1,6 Ångström liefert [16]. Ein Ångström (ein Zehntel Nanometer) entspricht etwa dem Durchmesser eines Atoms ( $\mathbf{A b b} \mathbf{8}$ ).

Damit hat man die Genauigkeit physikalischer Messmethoden erreicht (oder sogar überboten), die es erlaubt, von den räumlichen Strukturen auf Wirkungen zu schließen. Mit anderen Worten: Man braucht neue Medikamente nicht mehr suchen, indem man Tausende von Stoffen in ihrer Wirkung auf ein bestimmtes Protein im Labor untersucht, sondern indem man die Strukturen berechnet. Man kann sich auch die zeitraubenden röntgenkristallografischen Untersuchungen von Proteinen sparen, weil die neuronalen Netze wie AlphaFold 2 ebenso genau, aber um Größenordnungen (Tage anstatt Monate) schneller sind. Mit dieser „Protein-Revolution“ (im Hinblick auf deren Strukturaufklärung) dürfte ein „goldenes Zeitalter der strukturellen Biologie “ [1] anbrechen: Von der Sequenz her kennt man 180 Millionen Proteine, deren räumliche Gestalt man bislang in nur 170 Tausend Fällen experimentell untersuchte und damit bekannt machte $[2,36]$. Das wird sich jetzt ändern und zu

10 Das Levinthal-Paradoxon beruht darauf, dass die kombinatorische Vielzahl möglicher Faltungen eines Proteins, die mit der Länge der Aminosäurekette exponentiell zunimmt. Selbst wenn jeder Aminosäurerest nur 2 Zustände annehmen könnte, gäbe es bei einer Proteinlänge von n Aminosäuren $2^{\mathrm{n}}$ mögliche Faltungsvarianten. Nimmmt man an, dass sich das Protein im Verlauf seiner Translation am Ribosom faltet und nimmt man einen Zeitbedarf pro Faltung von 0,1 Pico-Sekunden $\left(10^{-13} \mathrm{~s}\right)$ an, dann würde die Faltung eines 150 Aminosäuren langen Proteins $2^{150} \mathrm{mal} 10^{-13} \mathrm{~s}$ dauern. Das ergibt mehr als $10^{24} \mathrm{~J}$ ahre, also knapp 100 Billionen $\left(10^{14}\right)$ mal länger als das Universum alt ist (ca. 12 Milliarden - also gut $10^{10}$ - Jahre). Tatsächlich aber nehmen Proteine ihre die gefaltete Form meist in Sekundenbruchteilen bis Minuten an und haben in dieser Form dann Halbwertszeiten von wenigen Stunden bis Tagen. Die Faltung kann daher definitiv nicht durch ein zufälliges Ausprobieren aller Möglichkeiten erklärt werden. Vielmehr muss es Mechanismen geben, die für eine rasche Faltung sorgen. In der Bioinformatik hat dieses Problem lange dafür gesorgt, dass man mathematische Lösungen der Proteinfaltung für unmöglich hielt. Dies änderte sich erst mit Deep Learning in großen neuronalen Netzwerken - vor wenigen Wochen.

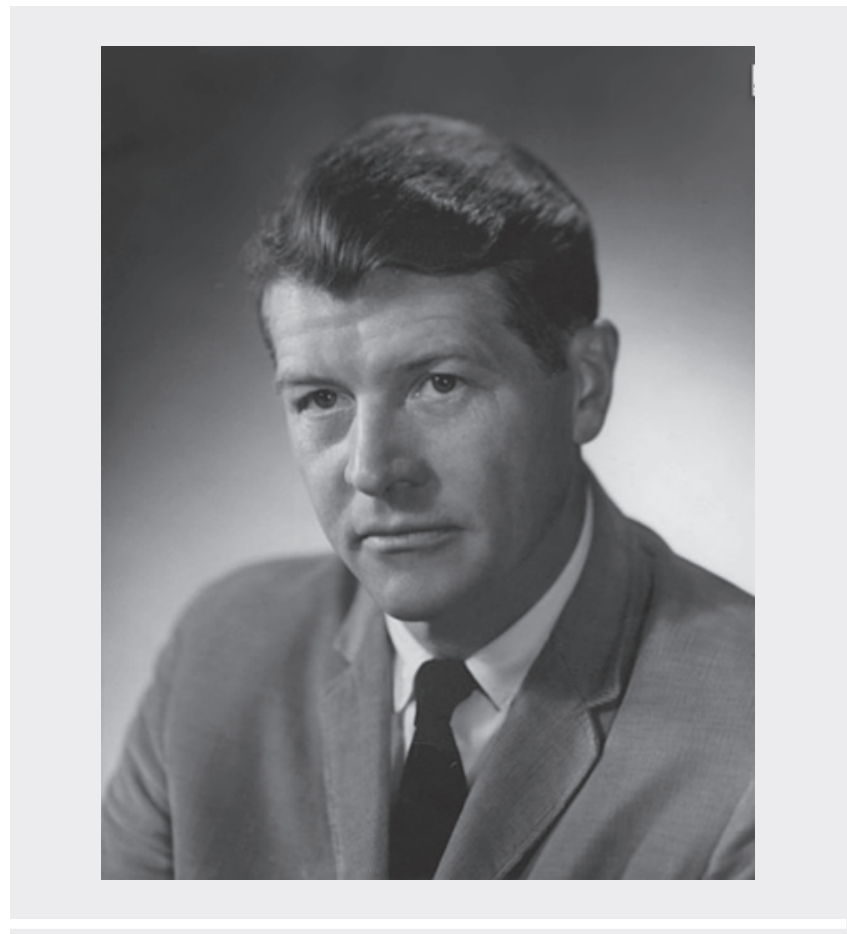

- Abb. 7 Der US-amerikanische Chemiker Christian B. Anfinsen (1916-1995) war Sohn norwegischer Einwanderer und erhielt 1972 den Nobelpreis für Chemie (Aufnahme von Edward A. Hubbard, National Institutes of Health, aus dem Jahr 1969; Foto: (๑) public domain).

einer explosionsartigen Vermehrung der Anzahl neuer Medikamente führen ${ }^{11}$ - auch in Psychiatrie und Neurologie.

Zurück also zur Nervenheilkunde. Vieles haben wir gelernt und verstehen z. B. Zustände wie Meditation, Flow oder Dissoziation mit Hilfe von Mausmodellen auf der Systemebene immer besser [23-34]. Aber noch viel mehr Neues wird uns erreichen: Wir können „kranke Gene“ sowie unsere Darmflora editieren, werden neue Muster psychopathologischer Gestalten durch die Möglichkeiten von Big Data finden. Und nicht zuletzt werden aufgrund der mittels neuronaler Netzwerke möglichen genauen Vorhersagen der Gestalten von Proteinen eine Fülle neuer Medikamente zu deren Beeinflussung hinzukommen. Das Fach Psychiatrie bleibt also spannend wie in den Jahrzehnten zuvor, wahrscheinlich wird es aber nochmals viel spannender.

11 Wer meine Ausführungen für übertrieben hält, den mögen die Ausführungen des Teams von AlphaFold vielleicht überzeugen: „As well as accelerating understanding of known diseases, we're excited about the potential for these techniques to explore the hundreds of millions of proteins we don't currently have models for - a vast terrain of unknown biology. Since DNA specifies the amino acid sequences that comprise protein structures, the genomics revolution has made it possible to read protein sequences from the natural world at massive scale - with 180 million protein sequences and counting in the Universal Protein database (UniProt). In contrast, given the experimental work needed to go from sequence to structure, only around 170,000 protein structures are in the Protein Data Bank (PDB). Among the undetermined proteins may be some with new and exciting functions and - just as a telescope helps us see deeper into the unknown universe - techniques like AlphaFold may help us find them" [36]. 

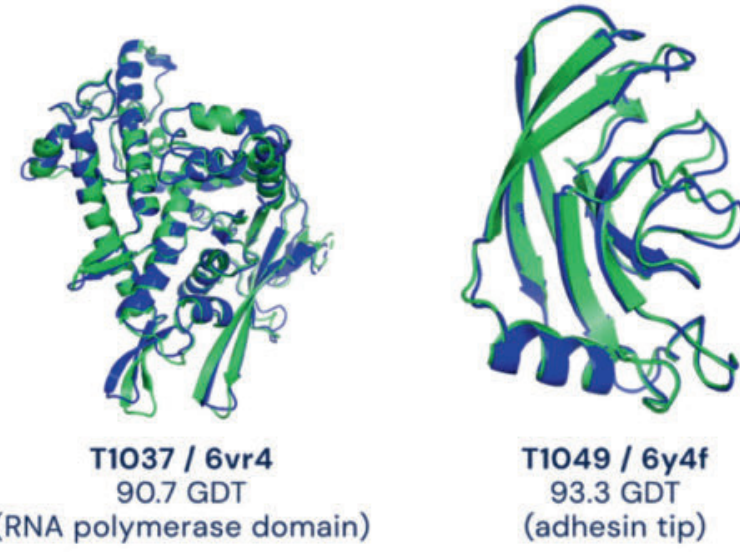

- Experimental result

Computational prediction

- Abb. 8 Screenshot zweier animierter räumlicher Proteinmodelle aus einem Blog vom 30.11.2020 des Teams von AlphaFold [36], der die Übereinstimmung von Experiment (grün) und Berechnung (blau) am Beispiel zweier Proteine darstellen soll.

Literatur

[1] AlQuraishi M. Protein-structure prediction gets real. Nature 2020; 577: 627-628

[2] Anonymus. The shape of things to come. DeepMind's latest Al could spark a medical revolution. New Scientist 2020: 248 (3311): 5

[3] Ardila D, Kiraly AP, Bharadwaj S, et al. End-to-end lung cancer screening with three-dimensional deep learning on low-dose chest computed tomography. Nature Medicine 2019; 25: 954-961

[4] Averina OV, Zorkina YA, Yunes RA, et al. Bacterial Metabolites of Human Gut Microbiota Correlating with Depression. International Journal of Molecular Sciences 2020; 21: 9234

[5] Bleuler E. Dementia Praecox oder die Gruppe der Schizophrenien. Leipzig: Franz Deuticke; 1911

[6] Chance FS, Aimone JB, Musuvathy SS, et al. Crossing the Cleft: Communication Challenges Between Neuroscience and Artificial Intelligence. Front Comput Neurosci 2020; 14: 39

[7] Esteva A, Kuprel B, Novoa RA, et al. Dermatologist-level classification of skin cancer with deep neural networks. Nature 2017; 547: 115-118

[8] Galton F. Composite portraits. Nature 1878; 18: 97-100

[9] Galton F. Composite portraits, made by combining those of many different persons into a single, resultant figure. Journal of the Anthropological Institute 1879; 8: 132-144

[10] Guyon A, Rousseau J, Bégin F-G, et al. Base editing strategy allows insertion of the A673T mutation in APP gene to prevent the development of Alzheimer's disease. bioRxiv 2020.10.27.357830

[11] Hassabis D, Kumaran D, Summerfield C, et al. Neuroscience-Inspired Artificial Intelligence. Neuron 2017; 95: 245-258

[12] Hebert JC, Radford-Smith DE, Probert F, et al. Mom's diet matters: Maternal prebiotic intake in mice reduces anxiety and alters brain gene expression and the fecal microbiome in offspring. Brain Behav Immun 2020; S0889-1591(20)31548-8
[13] Hermle L. Die Degenerationslehre in der Psychiatrie. Fortschr Neurol Psychiat 1986; 54: 69-79

[14] Howgego J. Al chemist figures out how to legally clone expensive patented drugs. New Scientist 2019; 3214, https://www.newscientist. com/article/mg24132143-500-ai-chemist-figures-out-how-to-legallyclone-expensive-patented-drugs/; abgerufen am 7.2.2019

[15] Jonsson T, Atwal JK, Steinberg S, et al. A mutation 404 in APP protects against Alzheimer's disease and age-related cognitive decline. Nature 2012; 488: 96-99

[16] Jumper ] et al. High Accuracy Protein Structure Prediction Using Deep Learning. In Fourteenth Critical Assessment of Techniques for Protein Structure Prediction Abstract Book 2020 https://predictioncenter.org/ casp14/doc/CASP14_Abstracts.pdf; abgerufen am 15.12.2020

[17] Kanjilal S, Oberst M, Boominathan S, et al. A decision algorithm to promote outpatient antimicrobial stewardship for uncomplicated urinary tract infection. Sci Transl Med 2020; 12

[18] Kraepelin E. Psychiatrie. Leipzig: Barth; 1899

[19] Kretschmer E. Körperbau und Charakter. Untersuchungen zum Konstitutionsproblem und zur Lehre von den Temperamenten. Berlin: Springer; 1977

[20] Lombroso C. Genio e follia; in deutscher Übersetzung: Genie und Irrsinn, erschienen. Stuttgart: Reclam; 1887

[21] Lu MT, Ivanov A, Mayrhofer T, et al. Deep Learning to Assess Long-term Mortality. From Chest Radiographs. JAMA Network Open 2019; 2 (7): e197416

[22] Malan-Muller S, Valles-Colomer M, Raes J, et al. The Gut Microbiome and Mental Health: Implications for Anxiety- and Trauma-Related Disorders. OMICS 2018; 22 (2): 90-107

[23] Martina Sgritta M, Dooling SW, Buffington SA, et al. Mechanisms Underlying Microbial-Mediated Changes in Social Behavior in Mouse Models of Autism Spectrum Disorder. Neuron 2019; 101: 246-259

[24] Reardon S. Rise of Robot Radiologists. Nature 2019; 576: S55-S58

[25] Rutledge R. Injury severity and probability of survival assessment in trauma patients using a predictive hierarchical network model derived from ICD-9 codes. J Trauma 1995; 38 (4): 590-601

[26] Schneider K. Klinische Psychopahologie. Stuttgart: Thieme; 1946/1980

[27] Senior AW, Evans R, Jumper ], et al. Improved protein structure prediction using potentials from deep learning. Nature 2020; 577: 706-710

[28] Silver $D$ et al. Mastering the game of Go with deep neural networks and tree search. Nature 2016; 529: 484-489

[29] Silver $D$ et al. Mastering the game of Go without human knowledge. Natur 2017; 550: 345-359

[30] Spitzer M, Hermle L. Von der Degeneration zur Antizipation: Systematische und wissenschaftliche Aspekte der Genetik neuro-psychiatrischer Erkrankungen. Nervenarzt 1995; 66: 187-196

[31] Spitzer M. Go und die Moral in Südkorea. Nervenheilkunde 2016; 35: 287-291

[32] Spitzer M. www (WeltWeite Werbung) und die Folgen. Nervenheilkunde 2018: 37: 303-311

[33] Spitzer M. Meditation, Mäuse und Myelin. Nervenheilkunde 2018; 37: 745-748

[34] Spitzer M. Psychotherapie im Mausmodell. Nervenheilkunde 2019; 38: 231-339

[35] Spitzer M. Dissoziation bei Mensch und Maus. Nervenheilkunde 2020; 39: $847-854$

[36] Tat, The AlphaFold team. AlphaFold: a solution to a 50-year-old grand challenge in biology. Blog Post Research, 30.11 .2020 https://deepmind.com/blog/article/alphafold-a-solution-to-a-50-year-old-grandchallenge-in-biology; abgerufen am 15.12.2020

[37] Valles-Colomer M, Falony G, Darzi Y, et al. Nat Microbiol 2019; 4 (4): 623-632 